\title{
Evaluation of Hepatoprotective Effect of Curcumin on Liver Cirrhosis Using a Combination of Biochemical Analysis and Magnetic Resonance-Based Electrical Conductivity Imaging
}

\author{
Eun Jung Kyung, ${ }^{1}$ Hyun Bum Kim, ${ }^{2}$ Eun Sang Hwang, ${ }^{2}$ Seok Lee, ${ }^{2}$ Bup Kyung Choi, ${ }^{3}$ \\ Jin Woong Kim $\left(\mathbb{0},{ }^{4}\right.$ Hyung Joong Kim $\left({ }^{3},{ }^{3}\right.$ Sang Moo Lim, ${ }^{5}$ Oh In Kwon, ${ }^{6}$ and Eung Je Woo $\oplus^{3}$ \\ ${ }^{1}$ College of Medicine, Chung-Ang University, Seoul 06974, Republic of Korea \\ ${ }^{2}$ Department of East-West Medical Science, Kyung Hee University, Yongin 17104, Republic of Korea \\ ${ }^{3}$ Impedance Imaging Research Center (IIRC), Kyung Hee University, Seoul 02447, Republic of Korea \\ ${ }^{4}$ Department of Radiology, Chonnam National University Medical School, Gwangju 61469, Republic of Korea \\ ${ }^{5}$ Department of Nuclear Medicine, Korea Institute of Radiological and Medical Sciences, Seoul 01812, Republic of Korea \\ ${ }^{6}$ Department of Mathematics, Konkuk University, Seoul 05029, Republic of Korea
}

Correspondence should be addressed to Jin Woong Kim; jw4249@hanmail.net and Hyung Joong Kim; bmekim@khu.ac.kr

Received 1 September 2017; Accepted 11 April 2018; Published 17 May 2018

Academic Editor: Mirella Giovarelli

Copyright (c) 2018 Eun Jung Kyung et al. This is an open access article distributed under the Creative Commons Attribution License, which permits unrestricted use, distribution, and reproduction in any medium, provided the original work is properly cited.

\begin{abstract}
In oriental medicine, curcumin is used to treat inflammatory diseases, and its anti-inflammatory effect has been reported in recent research. In this feasibility study, the hepatoprotective effect of curcumin was investigated using a rat liver cirrhosis model, which was induced with dimethylnitrosamine (DMN). Together with biochemical analysis, we used a magnetic resonance-based electrical conductivity imaging method to evaluate tissue conditions associated with a protective effect. The effects of curcumin treatment and lactulose treatment on liver cirrhosis were compared. Electrical conductivity images indicated that liver tissues damaged by DMN showed decreased conductivity compared with normal liver tissues. In contrast, cirrhotic liver tissues treated with curcumin or lactulose showed increased conductivity than tissues in the DMN-only group. Specifically, conductivity of cirrhotic liver after curcumin treatment was similar to that of normal liver tissues. Histological staining and immunohistochemical examination showed significant levels of attenuated fibrosis and decreased inflammatory response after both curcumin and lactulose treatments compared with damaged liver tissues by DMN. The conductivity imaging and biochemical examination results indicate that curcumin's anti-inflammatory effect can prevent the progression of irreversible liver dysfunction.
\end{abstract}

\section{Introduction}

Cirrhosis is histologically defined as an advanced fibrosis of liver tissues. Liver fibrosis is a hepatic response to chronic injury and is characterized by excess deposition of collagen, proteoglycans, and other macromolecules in the extracellular matrix [1]. These result in tissue degeneration and finally lead to portal hypertension and end-stage liver disease [1]. Histological changes from cirrhosis were considered irreversible, but recent studies have reported that liver fibrosis, even the more advanced stages, can be ameliorated with appropriate therapy [2]. The amelioration of liver fibrosis not only prevents the development of liver cirrhosis but can also reduce the incidence of hepatocellular carcinoma. Therefore, the appropriate therapy may help to extend the life span of patients with liver fibrosis from a long-term perspective.

Herbal medicine has received attention as a potential treatment for liver cirrhosis [3]. For example, extracts from Artemisia capillaris Thunb. reduced the production of fibrogenic factors, and a specific fragment inhibited fibroblast proliferation [4,5]. The Anemarrhena rhizome has been used for the treatment of inflammatory diseases such as lung disease, fever, and diabetes. Curcumin is a 
yellow pigment found in the rhizome of the spice turmeric (Curcuma longa Linn., a member of the Zingiberaceae family). Curcumin has antioxidant, anti-inflammatory, and anticarcinogenic pharmacological effects [6]. It acts by either interacting with molecular targets directly or altering gene expression and signaling pathways. Recent studies have reported that curcumin effectively inhibits liver cirrhosis through its action on many pathways; for example, it inhibits the NF- $\kappa \mathrm{B}$ pathway and reduces oxidative stress [7]. Thus, curcumin has potential as a therapy for liver diseases.

Imaging techniques are widely used to objectively visualize therapeutic effects, but there still exists demand for novel contrast information prior to morphological changes. Recent developments in magnetic resonance- (MR-) based tissue property mapping techniques have improved noninvasive imaging of electrical conductivity distribution inside living tissues [8]. Magnetic resonance electrical impedance tomography (MREIT) is a bioimaging modality that can provide high-resolution conductivity images based on the currentinjection MR method $[8,9]$. It is well known that the electrical conductivity of biological tissues is primarily determined by the concentration and mobility of ions which existed in the intra- and extracellular structures [10]. Electrical conductivity changes are closely related to the physiological and pathological conditions of tissues or organs [10-12]. Liver tissues mostly consist of hepatocytes, which make up $70 \sim 85 \%$ of the liver mass; therefore, the variations of ion concentration and mobility inside the liver may show similar patterns [10-12]. This suggests that liver tissues can exhibit uniform distribution of electrical tissue conductivity. Therefore, MREIT is a clinically useful method to image these features.

The purpose of this study was to evaluate the effects of curcumin on liver cirrhosis using an in vivo rat model. After induction of liver cirrhosis, we performed MR-based electrical conductivity imaging to visualize the tissue response after different treatments. The pathological state of the liver was evaluated using $\alpha$-smooth muscle actin ( $\alpha$-SMA) and cyclooxygenase-2 (COX-2) expression levels, and a biochemical marker of inflammation was used to estimate the functional state of liver tissues. The effects of curcumin treatment were compared with those of lactulose which is typically used as a positive effector on liver cirrhosis.

\section{Materials and Methods}

2.1. Animal Preparation and Drug Administration. A total of 32 Sprague-Dawley rats (8 weeks old, weighing 260 280 g) were used. The rats were fed on a standard pellet diet supplied by Orient Bio Inc. (Gyeonggi, South Korea) and housed two per cage at animal facilities. The temperature was maintained at $25 \pm 1{ }^{\circ} \mathrm{C}$, and relative humidity was $60 \pm 5 \%$. The rats were subject to a 12-hour light/dark cycle (lights on 8 a.m. and off 8 p.m.). All the rats were allowed to adapt to the laboratory conditions for 1 week before the experiments. During the experiments, they were given tap water and a standard diet ad libitum and weighed each week to evaluate their state of health. The rats were divided into the following four groups: normal control: intraperitoneal (IP) injection of phosphate-buffered saline (PBS) and oral administration of distilled water (DW); DMN-only: IP injection of $N$-nitrosodimethylamine (DMN, 48670, Sigma-Aldrich, St. Louis, MO, USA) and oral administration of DW; DMN with Lac: IP injection of DMN and oral administration of lactulose; and DMN with Cur: IP injection of DMN and oral administration of curcumin.

To induce liver cirrhosis, a dose of $10 \mu \mathrm{g} / \mathrm{kg}$ DMN was intraperitoneally injected twice a week for 4 weeks (dissolved in PBS). In the normal control group, PBS alone was injected in the same manner. All hepatoprotective oral treatments were conducted on the same day and for the same duration as the DMN and PBS injections referred to above. In the normal control and DMN-only groups (which received IP injections of $\mathrm{PBS}$ and $\mathrm{DMN}$, resp.), DW was orally administered. Curcumin (C1386, Sigma-Aldrich, St. Louis, MO, USA; $100 \mathrm{mg} / \mathrm{kg}$ ) and lactulose (644913501, Joong-Wae Pharma Co., Seocho, Seoul, Korea; $250 \mathrm{mg}$ / $\mathrm{kg}$ ) were orally administered to the groups that received IP injections of DMN. Curcumin was dissolved in $1 \%$ dimethyl sulfoxide at a concentration of $10 \mathrm{mg} / \mathrm{mL}$; lactulose was not diluted [13-16]. On the 6th week after administration, animals were sacrificed and their liver blocks were resected for the MREIT imaging experiments and for biochemical evaluations, such as tests of inflammatory indicator levels and staining data (Figures 1(a) and 1(b)). All animal procedures complied with the Institutional Care and Use Committee (KHUASP(SE)-15-024) of Kyung Hee University.

2.2. Phantom Preparation and Imaging Experiment. A cylindrical acrylic phantom $13 \mathrm{~cm}$ in diameter and $16 \mathrm{~cm}$ in height was used for the MREIT imaging experiment. The resected liver blocks containing the fibrotic lesion (Figure 1(b)) were positioned inside the center of the phantom. The phantom was filled with conductive material (agarose gel of $0.01 \mathrm{~S} / \mathrm{m}$ conductivity) to support the position of the liver block (Figure 1(c)). Four electrodes were attached to the sides of the phantom and imaging currents $I_{1}$ and $I_{2}$ were sequentially injected with two different directions through two pairs of opposing electrodes (Figure 1(d)). Following the phantom preparation time of over 30 minutes, the phantom was placed inside the bore of a 3T MRI scanner (Magnetom Trio A Tim, Siemens Medical Solutions, Erlangen, Germany). By this time, the phantom and liver blocks had reached thermal equilibrium at room temperature.

Using a constant current source, the first current $I_{1}$ was injected between one opposing pair of electrodes. The injected current was a $3 \mathrm{~mA}$ of amplitude and a total pulse width of $81 \mathrm{msec}$. After acquiring the first magnetic flux density $\left(B_{z}\right)$ data set for $I_{1}$, the second injection current $I_{2}$ with the same amplitude and pulse width was injected through the other pair of opposing electrodes to obtain the second data set. A spin-echo-based multiecho pulse sequence was used to obtain the MR magnitude and magnetic flux density $\left(B_{z}\right)$ images $[8,9]$. The imaging parameters for obtaining the conductivity images were as follows: repetition time/echo 


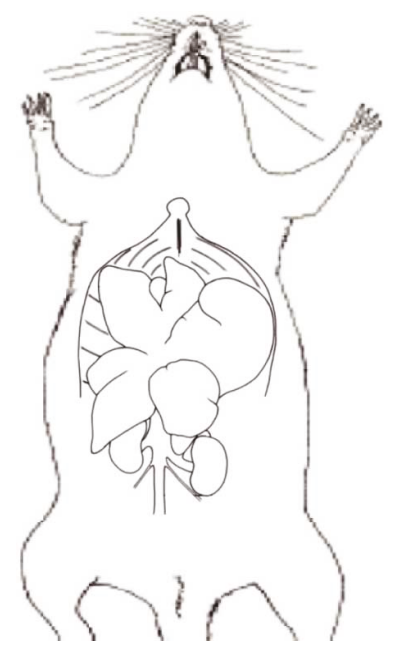

(a)

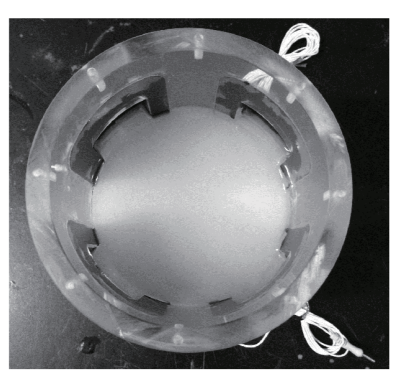

(c)

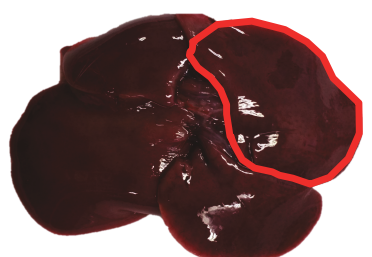

(b)

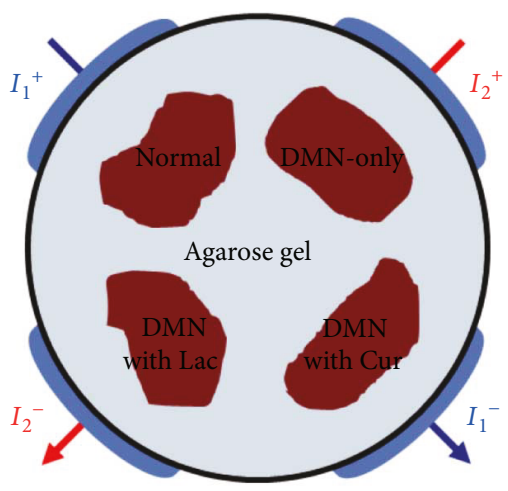

(d)

FIGURE 1: Experimental setup for MREIT conductivity imaging of liver phantom. (a) The whole liver was removed, and (b) the liver block was resected for phantom imaging. (c) A cylindrical acrylic phantom was filled with agarose gel of $1.0 \mathrm{~S} / \mathrm{m}$ conductivity to support the liver block. (d) Electrodes were attached to the sides of the phantom to inject imaging currents.

time $=1000 / 10,20,30$, and $40 \mathrm{msec}$ (four echoes), field of view $=160 \times 160 \mathrm{~mm}^{2}$, slice thickness $=3 \mathrm{~mm}$, number of excitations $=6$, matrix size $=128 \times 128$, and number of slices $=8$. The total imaging time to obtain the magnetic flux densities $\left(B_{\mathrm{z}, 1}\right.$ and $\left.B_{\mathrm{z}, 2}\right)$ by $I_{1}$ and $I_{2}$ was $25 \mathrm{~min}$. To reconstruct electrical tissue conductivity images, a single-step harmonic $B_{z}$ algorithm implemented in the software package CoReHA (conductivity reconstructor using harmonic algorithms) was used $[8,9]$. After conductivity image reconstruction, we measured the conductivities of whole liver blocks in the four different groups and analyzed the values.

2.3. HÆE Staining and Immunohistochemistry. Rats were anesthetized and transcardially perfusion-fixed with $4 \%$ formaldehyde in $0.1 \mathrm{M}$ sodium phosphate buffer ( $\mathrm{pH} 7.4$ ). Liver tissues were removed quickly and postfixed with the same fixation solution overnight at $4^{\circ} \mathrm{C} .20 \mu \mathrm{m}$ thick coronal sections of liver tissues were made using a freezing microtome (Leica, $2800 \mathrm{~N}$, Germany). The sections were stained with hematoxylin and eosin (H\&E) for tissue morphology observation. For immunohistochemical staining of $\alpha$-smooth muscle actin ( $\alpha$-SMA) and cyclooxygenase-2 (COX-2), free- floating liver sections were stained using 3,3' -diaminobenzidine $(\mathrm{DAB})$ reaction. The sections were rinsed with $0.05 \mathrm{M}$ PBS and incubated for $15 \mathrm{~min}$ in $1 \%$ hydrogen peroxide PBS at room temperature. The sections were incubated overnight at $4^{\circ} \mathrm{C}$ with primary antibody against $\alpha$-SMA (ab7817, Abcam, Cambridge, MA, USA, 1:2000 dilution) and COX2 (sc1747, Santa Cruz Biotech, Santa Cruz, CA, USA, $1: 1000$ dilution), then incubated with biotinylated anti-goat (sc2020, Santa Cruz Biotech, Santa Cruz, CA, USA, 1:200 dilution) and anti-mouse (sc2005, Santa Cruz Biotech, Santa Cruz, CA, USA, 1:200 dilution) secondary antibodies for 2 hours at room temperature, after which the avidin-biotin complex (Vector Laboratories, Burlingame, CA, USA) method was carried out with peroxidase coupling in a mixture containing $0.05 \%$ DAB (Sigma-Aldrich, St. Louis, MO, USA) and $0.03 \% \mathrm{H}_{2} \mathrm{O}_{2}$ for 2-5 min. Images of the DABcolorized brain sections were captured using a light microscope (BX51, Olympus, Tokyo, Japan) equipped with a CCD camera (DP70, Olympus).

2.4. Serum Biochemical Analysis. Blood was collected from the abdominal aorta on the final day of the experiment. After 
centrifuging at $3000 \times \mathrm{g}$ for $20 \mathrm{~min}$, the serum was separated and stored at $-70^{\circ} \mathrm{C}$. The serum levels of albumin (ALB), aspartate transaminase (AST), and alanine transaminase (ALT) were measured using an enzyme-linked immunosorbent assay (ELISA) kit. Samples and standards were analyzed using an auto chemistry analyzer (AU400, Olympus, Tokyo, Japan).

2.5. Statistical Analysis. The relative optical densities of various immunolabeled cells were measured and analyzed using ImageJ software (Ver. 1.44p, NIH, Bethesda, MD, USA). The relative optical densities were expressed by the mean gray values on an inverted black-white binary image and normalized against the values of the normal group with an area of $10^{5} \mu \mathrm{m}^{2}$. The mean values of four sections (obtained from the four groups: normal, DMN-only, DMN with Lac, and DMN with Cur) were statistically analyzed. The western blot results were converted to the percentage rate compared with the normal control, designated as $100 \%$. The converted values were used in the statistical analysis. We used a $t$-test to compare the difference between the two groups, using SPSS 20.0 for Windows (SPSS Inc., Chicago, IL, USA). The differences were considered statistically valid when the $p<0.05$.

\section{Results}

3.1. Body Weight Measurement. As the body weight change is one factor used to evaluate cirrhosis, we directly measured the body weight of all rats and compared the weight among the experimental groups (Table 1). During the experiments, body weight continually increased and was similar in all experimental groups over time, except the DMN-only group. The fluctuation in the DMN-only group may reflect tissue condition related to the degree of cirrhosis.

3.2. Serum Biochemical Assay Measurement. For serum biochemical analysis, we measured AST, ALT, and ALB levels, all of which are well-known indicators of liver functionality (Table 2). Comparison of normal and DMN-only groups showed that the AST and ALT levels in the DMN-only group were significantly higher than those in the normal control group, indicating the progression of liver injury. Comparison of treatment groups and the DMN-only group showed that the DMN with curcumin group exhibited significantly lower AST and ALT values than the DMN-only group, whereas the DMN with lactulose group exhibited significantly lower values only for ALT. There were no significant differences in ALB level among the experimental groups.

3.3. Electrical Conductivity of Liver Tissues. In order to visualize the hepatoprotective effect, we performed a MREIT conductivity imaging experiment using two liver tissue phantoms (Figure 2). Morphological information was obtained from the MR magnitude images (Figures 2(b) and 2(e)). There was no significant difference in contrast among the experimental groups. However, the conductivity images (Figure 2(c) and 2(f)) showed that the contrast was highest in normal liver tissue and lowest in DMN-only tissue. The conductivity contrast in both the treatment groups was
TABLE 1: Measurement of body weight in four experimental groups.

\begin{tabular}{lcccc}
\hline $\begin{array}{l}\text { Body weight } \\
(\mathrm{g})\end{array}$ & Normal & $\begin{array}{c}\text { DMN- } \\
\text { only }\end{array}$ & $\begin{array}{c}\text { DMN with } \\
\text { Lac }\end{array}$ & $\begin{array}{c}\text { DMN with } \\
\text { Cur }\end{array}$ \\
\hline Control & $259.7 \pm 2.0$ & $269.4 \pm 2.8$ & $265.9 \pm 2.5$ & $270.4 \pm 2.7$ \\
1 week & $317.7 \pm 7.0$ & $320.0 \pm 5.9$ & $325.0 \pm 4.9$ & $330.1 \pm 6.0$ \\
2 weeks & $367.0 \pm 6.2$ & $360.9 \pm 4.3$ & $367.4 \pm 1.8$ & $365.3 \pm 3.7$ \\
3 weeks & $372.9 \pm 6.7$ & $350.9 \pm 5.1$ & $394.6 \pm 9.1$ & $370.9 \pm 6.9$ \\
4 weeks & $409.7 \pm 8.4$ & $373.9 \pm 3.9$ & $396.9 \pm 8.7$ & $383.6 \pm 7.1$ \\
\hline
\end{tabular}

DMN: dimethylnitrosamine; Lac: lactulose; Cur: curcumin.

clearly higher than that in the DMN-only tissue. Specifically, the conductivity of DMN with curcumin was slightly higher than that of DMN with lactulose. The results from both phantoms showed a similar pattern.

For quantitative analysis, we measured the conductivity values of all liver tissues across experimental groups (Figure 3). The conductivity values were highest in the normal control and lower in the order of DMN with curcumin, DMN with lactulose, and DMN-only groups. The conductivity in the treatment groups was significantly higher than that in the DMN-only group. Therefore, it is possible to explain that curcumin has the hepatoprotective effects from the bioelectromagnetic standpoint.

3.4. Histopathological Findings. Liver tissues from each experimental group were subjected to $\mathrm{H} \& \mathrm{E}$ staining and their histological morphologies compared (Figure 4). The DMN-only group tissues exhibited histological abnormalities, such as congestion and destruction of hepatic architecture, massive and severe hepatocyte necrosis, and remarked mononuclear cell infiltration. In contrast, the severity of histological abnormalities and cirrhotic liver alterations in the lactulose and curcumin treatment groups was significantly lower than that in the DMN-only group. The size and number of abnormalities in the cirrhotic liver were lower in the curcumin treatment group than in the lactulose treatment group.

3.5. Effects on $\alpha$-SMA Expression. To evaluate hepatic stellate cell (HSC) activation in the experimental groups, $\alpha$-SMA expression was compared using immunohistochemical staining (Figure 5). All experimental groups showed increased $\alpha$-SMA signals compared with the normal control (Figure 5(a)). The highest expression of $\alpha$-SMA in the DMN-only group represents the highest extent of liver injury. Compared with the DMN-only group, the lactulose and curcumin treatment groups showed reduced $\alpha$-SMA expression. The $\alpha$-SMA expression level was numerically compared by counting the number of cells showing positive expression versus the total cell count (Figure 5(b)). The level of $\alpha$-SMA expression was highest in the DMNonly group. Compared with the DMN-only group, the curcumin treatment group showed significantly lower $\alpha$ SMA expression than the lactulose treatment group.

3.6. Effects on COX-2 Expression. The degree of inflammation among the experimental groups was compared using the 
TABLE 2: Measurement of serum biochemical parameters in four liver tissues.

\begin{tabular}{lcccc}
\hline Parameter & Normal & DMN-only & DMN with Lac & DMN with Cur \\
\hline AST (IU/L) & $78.67 \pm 15.69$ & $128.33 \pm 21.57^{*}$ & $106.00 \pm 37.24$ & $72.33 \pm 21.16^{\#}$ \\
ALT (IU/L) & $42.33 \pm 6.11$ & $67.33 \pm 8.73^{*}$ & $45.00 \pm 6.00^{\# \#}$ & $38.67 \pm 3.22^{\#}$ \\
ALB (g/dL) & $3.93 \pm 0.54$ & $3.81 \pm 0.77$ & $3.70 \pm 0.35$ & $3.75 \pm 0.63$ \\
\hline
\end{tabular}

Data are represented by mean \pm SEM ( $n=6$ in each group). Statistical significances are compared between normal versus DMN-only $\left({ }^{*} p<0.05\right)$, DMN-only versus DMN with Lac and with Cur $\left({ }^{\#} p<0.05 ; \# \#<0.01\right.$, resp.).

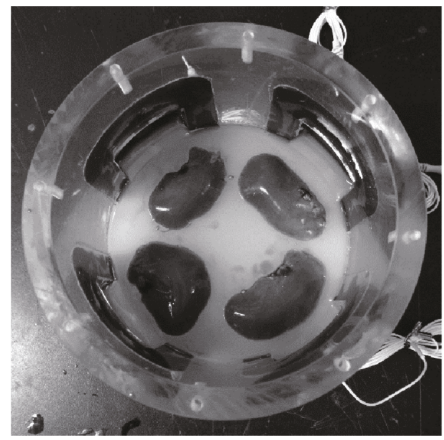

(a)

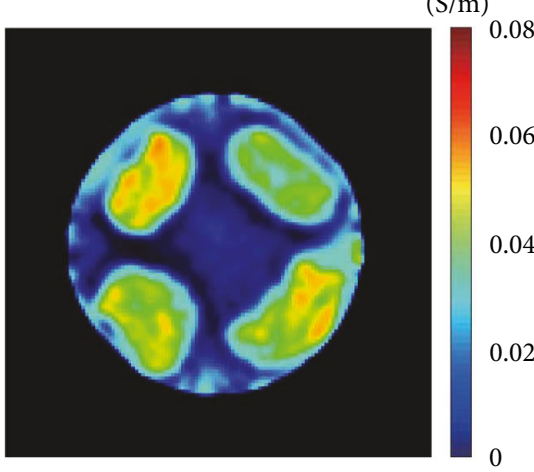

(c)

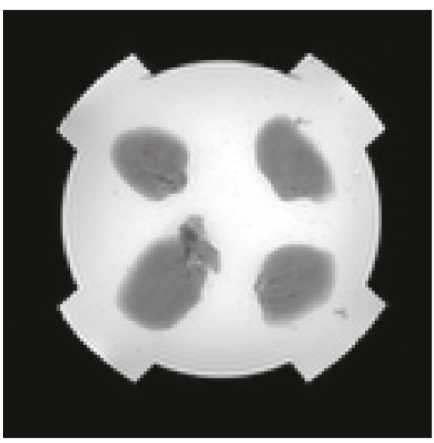

(e)

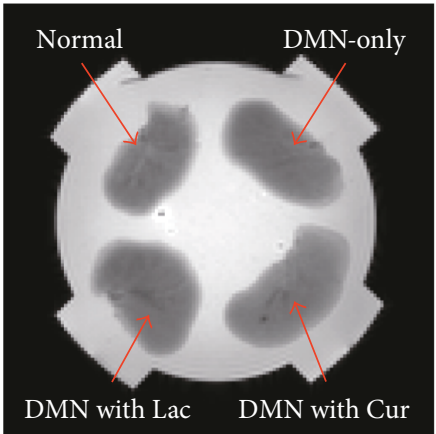

(b)

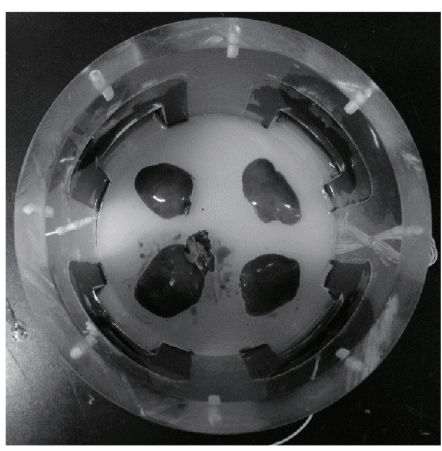

(d)

$(\mathrm{S} / \mathrm{m})$

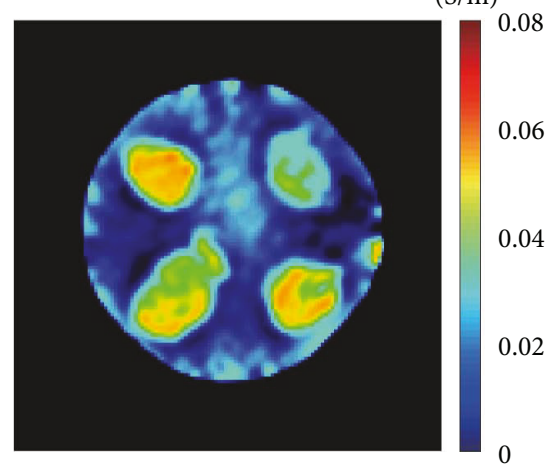

(f)

FIgure 2: Typical MREIT images of two liver phantoms. (a and d) Phantom consisted of four liver blocks including normal control, DMNonly, DMN with lactulose, and DMN with curcumin. (b and e) MR magnitude images provide morphological information on the liver phantom. (c and f) Electrical conductivity images of liver tissue indicate contrast information depending on their tissue condition.

level of COX-2 expression (Figure 6). The DMN-only group showed the highest COX-2 expression, indicating a successful induction of the fibrotic liver model (Figure 6(a)). Curcumin and lactulose treatment of the fibrotic liver had a mitigating effect on liver inflammation, as demonstrated by the decreased COX-2 expression in Figure 6(a). The COX-2 expression level was numerically compared by counting the number of cells showing positive expression versus the total 


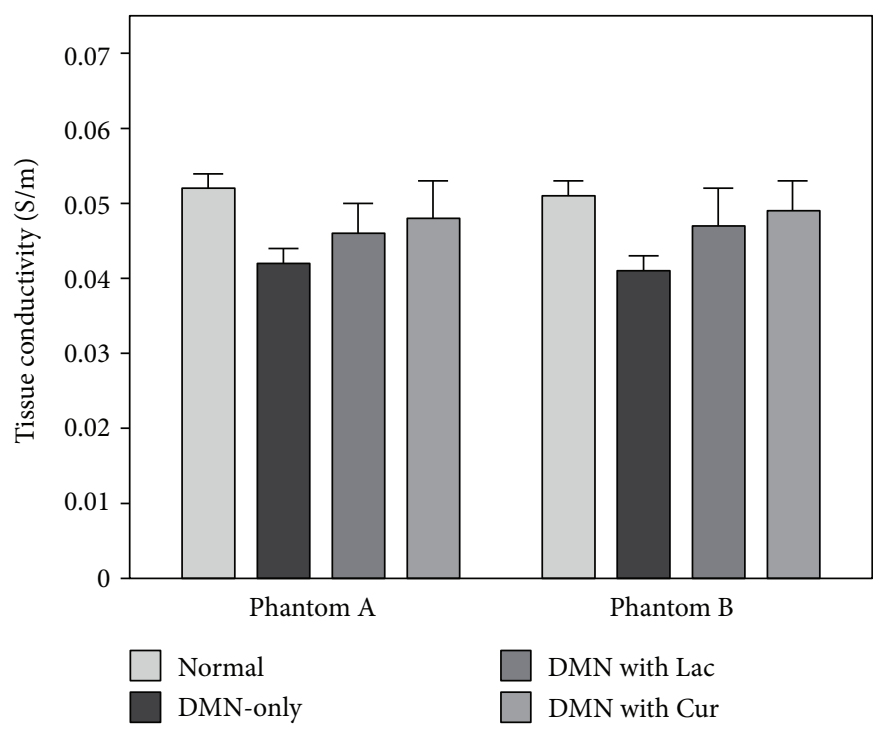

FIGURE 3: Bar graph showing quantitative analysis of conductivity contrast at four different experimental groups.

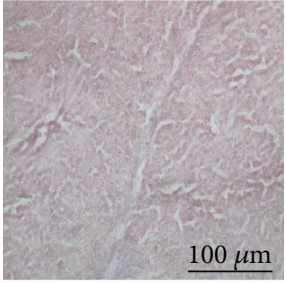

Normal

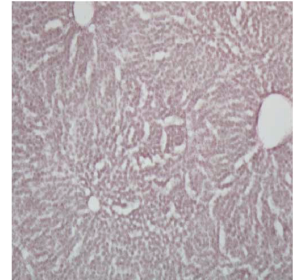

DMN

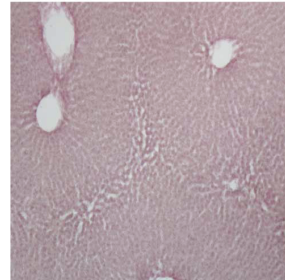

DMN with Lac

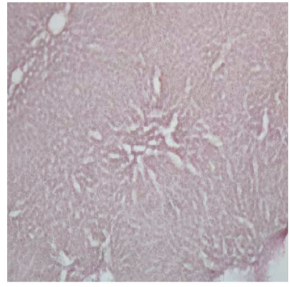

DMN with Cur

FIGURE 4: Histological observation of fibrotic liver tissues after curcumin and lactulose treatment. Sections obtained by frozen section were subjected to H\&E staining. DMN (10 mg/kg) or sterile saline was injected into the rat peritoneum three times per week for 4 weeks. A solution of curcumin, lactulose, or normal saline was given daily through oral administration for 28 days.

cell count (Figure 6(b)). The COX-2 expression level was highest in the DMN-only group. Compared with the DMNonly group, the lactulose and curcumin treatment groups showed significantly reduced COX-2 expression. The cell count in the DMN with curcumin group was slightly higher than that in the DMN with lactulose group, suggesting that curcumin also has a similar effect on the recovery of hepatic fibrosis as much as lactulose.

\section{Discussion}

In this study, we used DMN to induce liver cirrhosis to examine the ameliorating effect of herbal extracts on hepatic fibrosis. The general causes of chronic liver injuries and cirrhosis are alcohol, drugs, infections, autoimmune disease, vascular and metabolic disorders, biliary obstruction, and cryptogenic factors [17]. In rats, DMN induces broad micronodular cirrhosis with regenerative hepatocyte changes and bile duct proliferation. Phenomena such as increased mortality, destroyed hepatic parenchymal cells, and increased fibrotic tissue formation exhibited by DMNtreated rats are similar to the disease progression shown in human liver cirrhosis [18].

Histology of cirrhosis is typically characterized by diffuse fibrosis, regenerative nodules, and microvascular rearrangement [19]. The continuous hepatic damage can lead to fibrosis and cirrhosis, resulting in the hepatocytes eventually becoming surrounded by fibrous tissues. From a bioelectromagnetic perspective, this phenomenon is closely related to tissue conditions, including cellular structure, concentration and mobility of ions in intra- and extracellular structures, and other related factors [8-10]. At the initial stage of fibrosis, conductivity may increase owing to the excessive deposition of the extracellular matrix. Depending on the degree of fibrosis progression, conductivity will decrease owing to the damaged tissues, anisotropy, and liver stiffness of fibrosis [11, 12]. Electrical conductivity shows frequency-dependent spectra, so its values at different frequencies can provide different information. In this study, the MR-based electrical conductivity imaging method provided low-frequency conductivity information using externally injected currents. The advantage of low-frequency conductivity is that it provides information about cell membrane effects and tissue anisotropy according to the degree of cirrhosis. Several studies have reported that electrical conductivity provides unique information on tissue changes such as tumors, edema, ischemia, and liver fibrosis $[9,11,12]$.

Our MREIT conductivity images of liver phantoms provide information about tissue condition by treatment effect. 


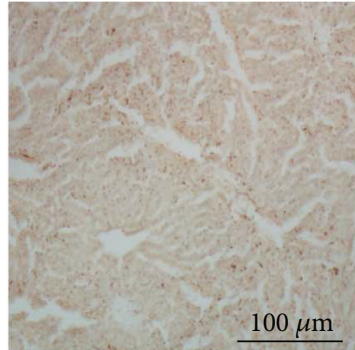

Normal

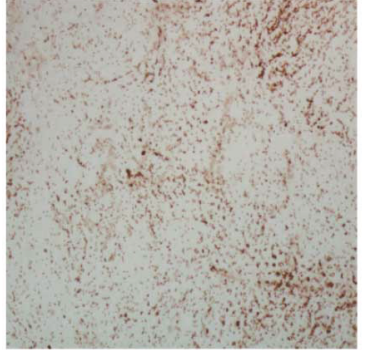

DMN-only

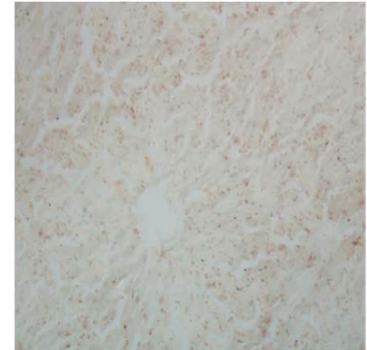

DMN with Lac

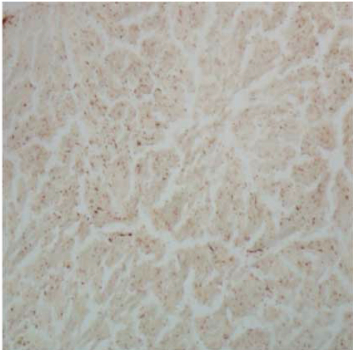

DMN with Cur

(a)

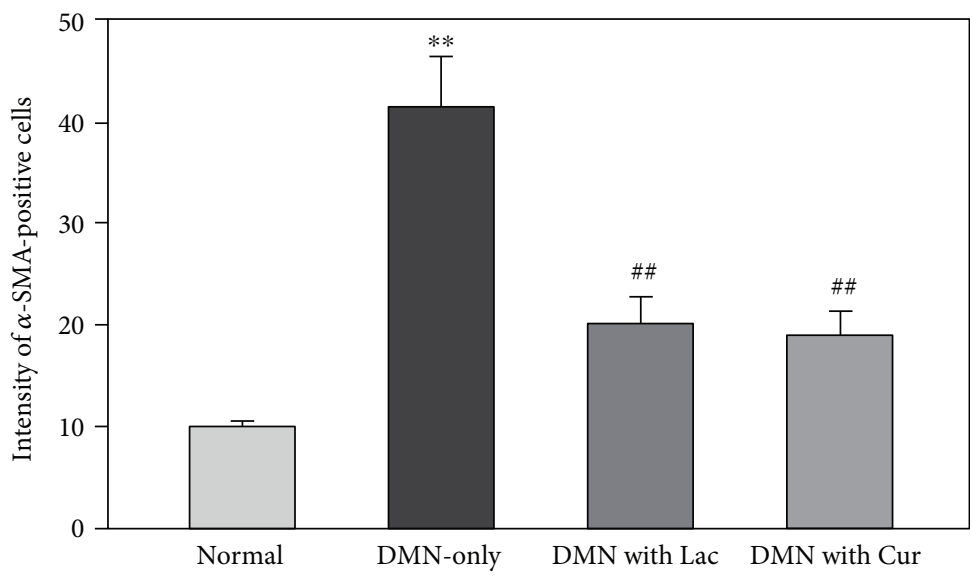

(b)

FIGURE 5: Effect of curcumin on $\alpha$-SMA expression in liver tissue. (a) Representative photographs showing immunostained $\alpha$-SMA in rat liver. (b) Positive cell count showing expression of $\alpha$-SMA in rat liver tissue. Data are represented by mean \pm SEM ( $n=6$ in each group). Statistical significances are compared between the normal and DMN-only groups $\left({ }^{* *} p<0.01\right)$ or between the DMN-only and DMN with treatment groups $\left({ }^{\#} p<0.01\right)$.

In the process of tissue damage by DMN injection, the movement and concentration of body fluids in the intra- and extracellular structure may influence the electrical conductivity. The lowest conductivity in the $\mathrm{DMN}$-only group indicates relatively severe tissue damage caused by a substance toxic to the liver. The increased conductivities in the two treatment groups with lactulose or curcumin suggest a hepatoprotective effect on liver cirrhosis. The higher conductivity in the DMN with curcumin group compared with the lactulose treatment group indicates a more powerful anticirrhotic effect on the liver. In addition, the electrical tissue conductivity images correlated with the biochemical analysis results.

Chronic liver injuries cause apoptosis, and this results in compensatory regeneration of hepatocytes [20]. Injured hepatocytes release fibrogenic mediators and reactive oxygen species, induce HSC activation, and stimulate fibrogenesis [21]. In the normal liver, HSCs located between hepatocytes and sinusoidal endothelial cells are quiescent. However, in response to liver injuries, HSCs are transformed to active contractile myofibroblasts and contribute to microcirculation disturbances $[22,23]$. Increases in HSC mobility and migration cause a process of sinusoidal remodeling [24], and excessive HSC function results in pericytic dysfunction, which is indicated by phenotypic markers such as $\alpha$-SMA [25]. Curcumin induces apoptosis and suppresses proliferation in HSCs. In addition, it inhibits extracellular matrix formation by enhancing HSC matrix metalloproteinase expression via peroxisome proliferatoractivated receptor gamma and by suppressing connective tissue growth factor expression [26]. Curcumin function in reducing HSC activation was confirmed by downregulation of $\alpha$-SMA. COX-2 is expressed in macrophages and cancer cells and involved in situations such as acute and chronic inflammation, hemodynamics, tumorigenesis, renal function, and hepatic fibrogenesis [23, 27]. Whereas activated HSCs turn into myofibroblasts and express COX-2, isolated HSCs do not express COX-2 [28]. The hepatoprotective effect of curcumin on liver cirrhosis, which showed reduced hepatic inflammation and fibrogenesis, was supported by the finding that the increased COX-2 expression in the DMN-only treatment was significantly reduced by curcumin treatment.

In conclusion, the use of herbal medicine might be helpful in preventing liver fibrosis. Our results indicate that curcumin ameliorated liver cirrhosis via its anti-inflammatory effect and suppression of HSC activity, thereby attenuating fibrosis. Together with biochemical analysis, we used a MR-based electrical conductivity imaging method to enhance the detection of curcumin's hepatoprotective effect. Both the analysis of biochemical parameters and 


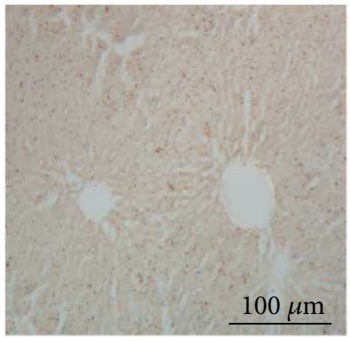

Normal

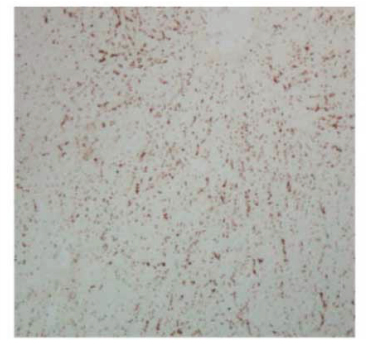

DMN-only

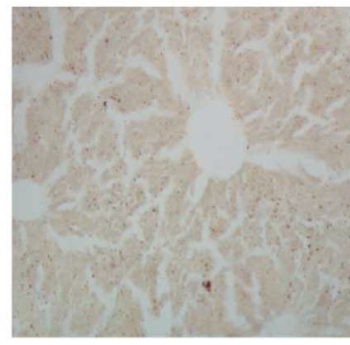

DMN with Lac

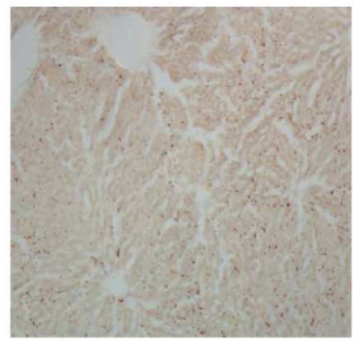

DMN with Cur

(a)

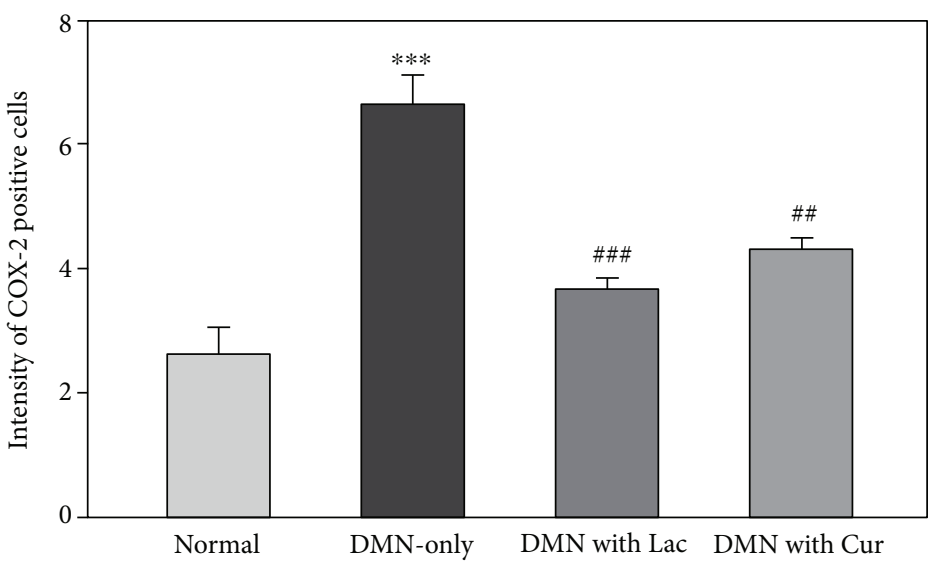

(b)

Figure 6: Effect of curcumin on COX-2 expression in liver tissue. (a) Representative photographs showing immunostained COX-2 in rat liver. (b) Positive cell count showing expression of COX-2 in rat liver tissue. Data are represented by mean \pm SEM ( $n=6$ in each group). Statistical significances are compared between the normal and DMN-only groups $\left({ }^{* * *} p<0.001\right)$ or between the DMN-only and DMN with treatment groups ( ${ }^{\# \#} p<0.01$; $\left.{ }^{\# \#} p<0.001\right)$.

the comparison of electrical tissue conductivity showed that the effects of curcumin were comparable to those of lactulose. Future studies should address the mechanism of curcumin's antifibrotic effect and conduct in vivo imaging experiments to further investigate its clinical usefulness as a hepatoprotective treatment.

\section{Conflicts of Interest}

The authors declare that there is no conflict of interest regarding the publication of this article.

\section{Authors' Contributions}

Eun Jung Kyung and Hyun Bum Kim contributed equally to this work.

\section{Acknowledgments}

This work was supported by the National Research Foundation of Korea (NRF) and Korea Institute of Radiological and Medical Sciences (KIRAMS) grants funded by the Korea Government (nos. 2015R1D1A1A09058104, 2017R1D1A3B04033613, 2017R1A2A1A05001330, and 50461-2018).

\section{References}

[1] D. Schuppan and N. H. Afdhal, "Liver cirrhosis," The Lancet, vol. 371, no. 9615, pp. 838-851, 2008.

[2] M. H. Ismail and M. Pinzani, "Reversal of liver fibrosis," The Saudi Journal of Gastroenterology, vol. 15, no. 1, pp. 72-79, 2009.

[3] J. J. Kim, J. Choi, M. K. Lee et al., "Immunomodulatory and antidiabetic effects of a new herbal preparation (HemoHIM) on streptozotocin-induced diabetic mice," Evidence-based Complementary and Alternative Medicine, vol. 2014, Article ID 461685, 8 pages, 2014.

[4] J. M. Han, H. G. Kim, M. K. Choi et al., “Artemisia capillaris extract protects against bile duct ligation-induced liver fibrosis in rats," Experimental and Toxicologic Pathology, vol. 65, no. 6, pp. 837-844, 2013.

[5] Y. Hong, M. Y. Kim, and M. Yoon, "The anti-angiogenic herbal extracts Ob-X from Morus alba, Melissa officinalis, and Artemisia capillaris suppresses adipogenesis in 3T3-L1 adipocytes," Pharmaceutical Biology, vol. 49, no. 8, pp. 775783, 2011.

[6] P. Qiu, J. Sun, S. Man et al., "Curcumin attenuates Nnitrosodiethylamine-induced liver injury in mice by utilizing the method of metabonomics," Journal of Agricultural and Food Chemistry, vol. 65, no. 9, pp. 2000-2007, 2017.

[7] Y. Cai, D. Lu, Y. Zou et al., "Curcumin protects against intestinal origin endotoxemia in rat liver cirrhosis by targeting 
PCSK9," Journal of Food Science, vol. 82, no. 3, pp. 772-780, 2017.

[8] E. J. Woo and J. K. Seo, "Magnetic resonance electrical impedance tomography (MREIT) for high-resolution conductivity imaging," Physiological Measurement, vol. 29, no. 10, pp. R126, 2008.

[9] T. I. Oh, W. C. Jeong, A. McEwan et al., "Feasibility of magnetic resonance electrical impedance tomography (MREIT) conductivity imaging to evaluate brain abscess lesion: in vivo canine model," Journal of Magnetic Resonance Imaging, vol. 38, no. 1, pp. 189-197, 2013.

[10] S. Grimnes and O. G. Martinsen, Bioimpedance and Bioelectricity Basics , Academic Press, London, UK, 2000.

[11] D. Haemmerich, D. J. Schutt, A. S. Wright, J. G. Webster, and D. M. Mahvi, "Electrical conductivity measurement of excised human metastatic liver tumours before and after thermal ablation," Physiological Measurement, vol. 30, no. 5, pp. 459-466, 2009.

[12] D. Haemmerich, S. T. Staelin, J. Z. Tsai, S. Tungjitkusolmun, D. M. Mahvi, and J. G. Webster, "In vivo electrical conductivity of hepatic tumours," Physiological Measurement, vol. 24, no. 2, pp. 251-260, 2003.

[13] J. Yu, W. Zhang, R. Zhang, X. Ruan, P. Ren, and B. Lu, "Lactulose accelerates liver regeneration in rats by inducing hydrogen," Journal of Surgical Research, vol. 195, no. 1, pp. 128-135, 2015.

[14] B. Taskin, M. A. Erdoğan, G. Yiğittürk, D. Günenc, and O. Erbaş, "Antifibrotic effect of lactulose on a methotrexateinduced liver injury model," Gastroenterology Research and Practice, vol. 2017, Article ID 7942531, 5 pages, 2017.

[15] F. B. Kasravi, D. Adawi, G. Molin, S. Bengmark, and B. Jeppsson, "Effect of oral supplementation of lactobacilli on bacterial translocation in acute liver injury induced by D-galactosamine," Journal of Hepatology, vol. 26, no. 2, pp. 417-424, 1997.

[16] O. Gozeneli, F. Tatli, A. E. Gunes et al., "Effects of thymoquinone and curcumin on the regeneration of rat livers subject to $70 \%$ hepatectomy," Acta Cirurgica Brasileira, vol. 33, no. 2, pp. 110-116, 2018.

[17] V. Cantisani, E. David, F. M. Meloni et al., "Recall strategies for patients found to have a nodule in cirrhosis: is there still a role for CEUS?," Medical Ultrasonography., vol. 17, no. 4, pp. 515$520,2015$.

[18] K. W. Kang, Y. G. Kim, M. K. Cho et al., "Oltipraz regenerates cirrhotic liver through CCAAT/enhancer binding proteinmediated stellate cell inactivation," The Faseb Journal, vol. 16, no. 14, pp. 1988-1990, 2002.

[19] N. P. Karidis, I. Delladetsima, and S. Theocharis, "Hepatocyte turnover in chronic HCV-induced liver injury and cirrhosis," Gastroenterology Research and Practice, vol. 2015, Article ID 654105, 8 pages, 2015.

[20] J. M. Schattenberg, M. Nagel, Y. O. Kim et al., "Increased hepatic fibrosis and JNK2-dependent liver injury in mice exhibiting hepatocyte-specific deletion of cFLIP," American Journal of Physiology - Gastrointestinal and Liver Physiology, vol. 303, no. 4, pp. G498-G506, 2012.

[21] R. Bataller and D. A. Brenner, "Liver fibrosis," The Journal of Clinical Investigation, vol. 115, no. 2, pp. 209-218, 2005.

[22] D. V. Garbuzenko, N. O. Arefyev, and D. V. Belov, "Mechanisms of adaptation of the hepatic vasculature to the deteriorating conditions of blood circulation in liver cirrhosis," World Journal of Hepatology, vol. 8, no. 16, pp. 665-672, 2016.

[23] K. Q. Hu, "Cyclooxygenase 2 (COX2)-prostanoid pathway and liver diseases," Prostaglandins, Leukotrienes, and Essential Fatty Acids, vol. 69, no. 5, pp. 329-337, 2003.

[24] J. S. Lee, D. Semela, J. Iredale, and V. H. Shah, "Sinusoidal remodeling and angiogenesis: a new function for the liverspecific pericyte?," Hepatology, vol. 45, no. 3, pp. 817-825, 2007.

[25] C. Hellerbrand, "Hepatic stellate cells-the pericytes in the liver," Pflügers Archiv - European Journal of Physiology, vol. 465, no. 6, pp. 775-778, 2013.

[26] M. A. O'Connell and S. A. Rushworth, "Curcumin: potential for hepatic fibrosis therapy?," British Journal of Pharmacology, vol. 153, no. 3, pp. 403-405, 2008.

[27] A. Juasook, T. Boonmars, Z. Wu et al., "Immunosuppressive prednisolone enhances early cholangiocarcinoma in Syrian hamsters with liver fluke infection and administration of N-nitrosodimethylamine," Pathology \& Oncology Research, vol. 19, no. 1, pp. 55-62, 2013.

[28] E. Efsen, A. Bonacchi, S. Pastacaldi et al., "Agonist-specific regulation of monocyte chemoattractant protein-1 expression by cyclooxygenase metabolites in hepatic stellate cells," Нераtology, vol. 33, no. 3, pp. 713-721, 2001. 


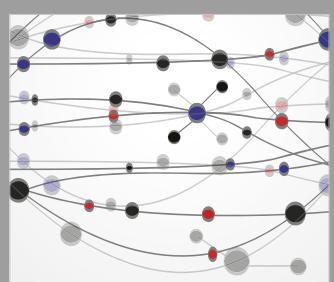

The Scientific World Journal
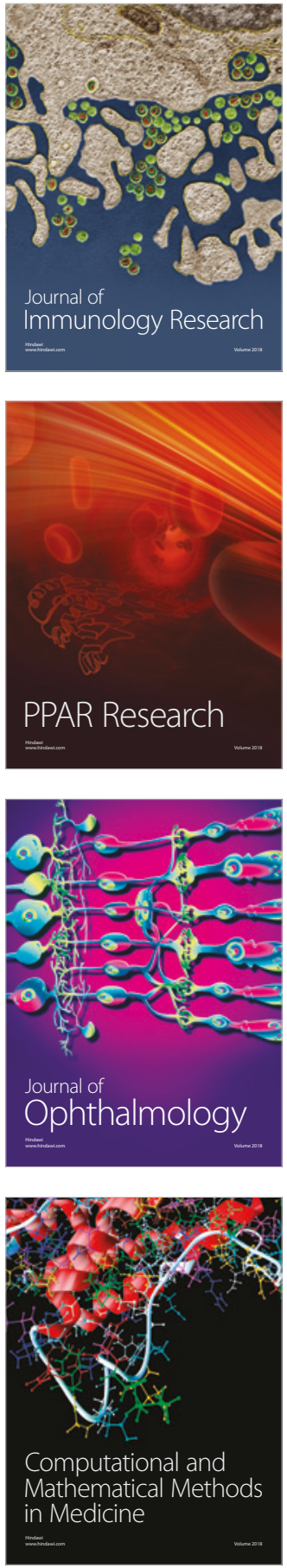

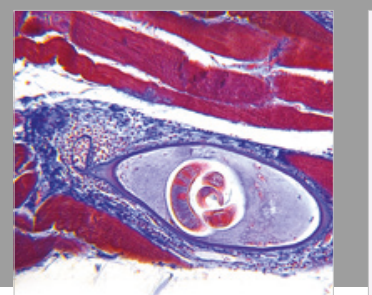

Gastroenterology Research and Practice

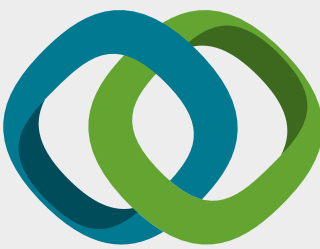

\section{Hindawi}

Submit your manuscripts at

www.hindawi.com
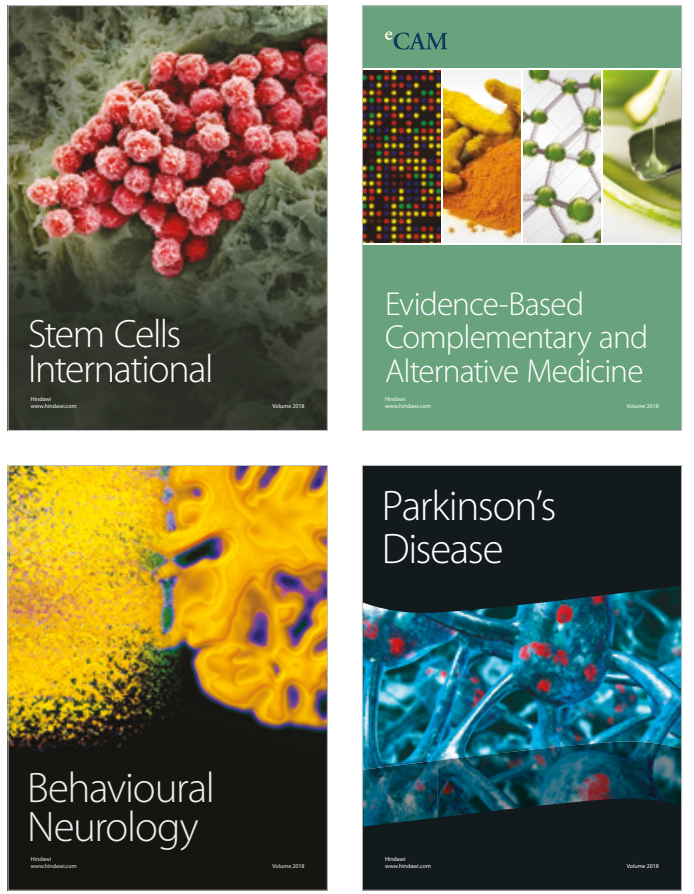

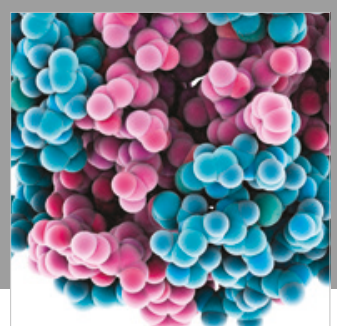

ournal of

Diabetes Research

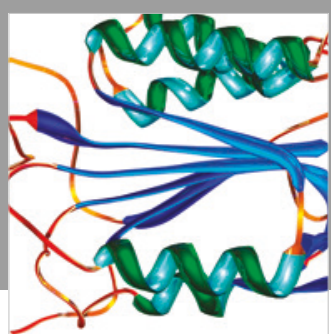

Disease Markers
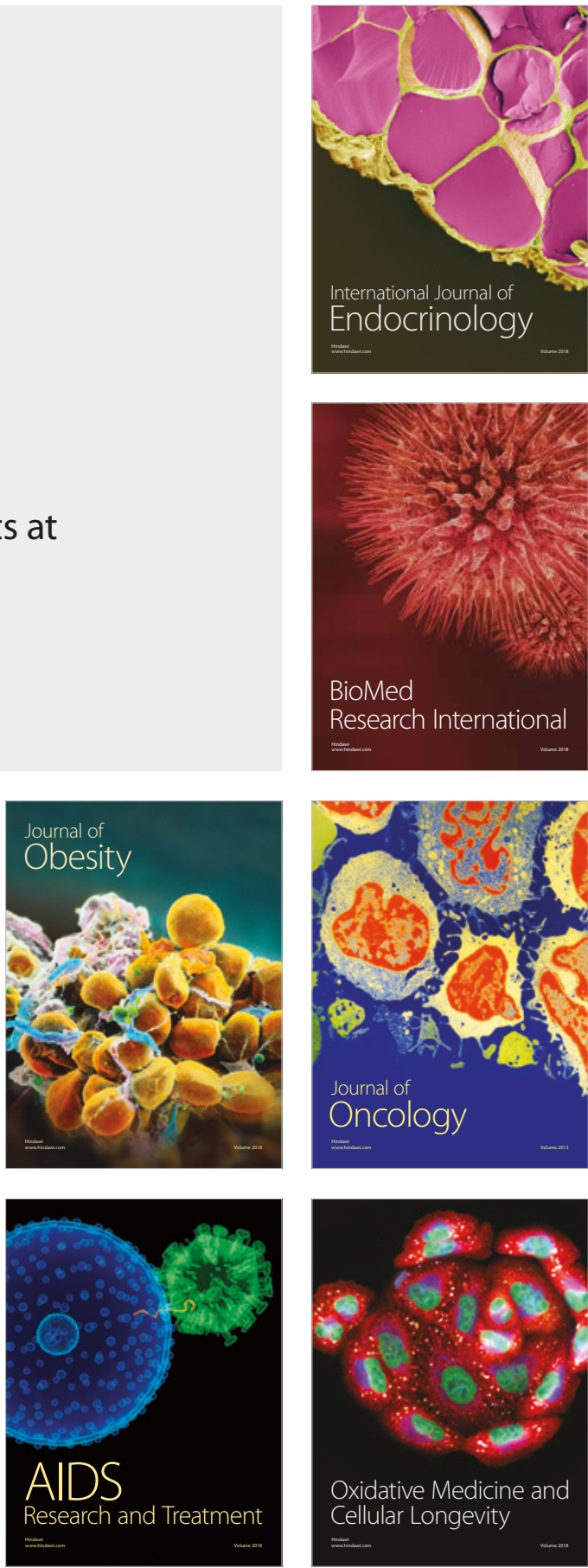